\title{
Frequency spectrum of the rapidly-oscillating mass-accreting component of the Algol-type system AS Eri
}

\author{
D. E. Mkrtichian ${ }^{1,2}$, A. V. Kusakinn ${ }^{3,4,10}$, E. Rodriguez ${ }^{5}$, A. Yu. Gamarova ${ }^{2,6}$, C. Kim ${ }^{7}$, S.-L. Kim ${ }^{8}$, \\ J. W. Lee ${ }^{8}$, J.-H. Youn ${ }^{8}$, Y. W. Kang ${ }^{1}$, E. C. Olson ${ }^{9}$, and K. Grankin ${ }^{11,12}$ \\ 1 Astrophysical Research Center for the Structure and Evolution of the Cosmos, 98 Gunja-Dong Gwangjin-Gu, \\ Sejong University, Seoul 143-747, Korea \\ 2 Astronomical Observatory, Odessa National University, Shevchenko Park, Odessa 65014, Ukraine \\ 3 Sternberg State Astronomical Institute, Universitetsky prospect 13, Moscow 119899, Russia \\ ${ }^{4}$ Fesenkov Astrophysical Institute of the Kazakhstan National Academy of Sciences, Kamenskoye plato, \\ Almaty 480068, Kazakhstan \\ 5 Instituto de Astrofisica de Andalucia, CSIC, PO Box 3004, 18080 Granada, Spain \\ 6 Thüringer Landessternwarte, 07778 Tautenburg, Germany \\ e-mail: anastasia@tls-tautenburg.de \\ 7 Department of Earth Science Education, Chonbuk National University, Chonju 516-756, Korea \\ 8 Korea Astronomy Observatory, Daejeon 305-348, Korea \\ 9 Astronomy Department, University of Illinois, 1002 W.Green St., Urbana, Illinois 61801, USA \\ ${ }^{10}$ Ulugh Beg Astronomical Institute, Astronomicheskaya St. 33, Tashkent 700052, Uzbekistan \\ 11 Isaac Newton Institute in Chile, Kazakhstan Branch, Russie \\ 12 Isaac Newton Institute in Chile, Uzbekistan Branch, Russie
}

Received 27 November 2002 / Accepted 15 January 2004

\begin{abstract}
The first multisite photometric campaign devoted to the rapidly oscillating mass-accreting (primary) component of the Algol-type eclipsing binary system AS Eri has confirmed the presence of rapid pulsations with frequency $59.03116 \mathrm{~d}^{-1}$, and revealed the second and third oscillation modes with frequencies $62.5631 \mathrm{~d}^{-1}$ and $61.6743 \mathrm{~d}^{-1}$, respectively. These modes are related to the 5-6 overtone oscillations and are among the shortest periods excited in non-magnetic MS A-F stars. The nearly equator-on visibility of eclipsing binaries help to narrow the range of possible mode identifications for the detectable modes as radial or $(l, m)=(1, \pm 1),(l, m)=(2, \pm 2)$ and $(l, m)=(2, \pm 0)$. We checked the high-order pulsation-to-orbital synchronization (POS) using the trial mode identification and the Doppler effect correction for frequencies of non-radial pulsation. We found that $(l, m, n)=(1,1,5)$ or $(2,2,5)$ and $(l, m, n)=(2,-2,6)$ identifications for $f_{1}$ and $f_{2}$ modes respectively satisfied the highorder POS. These mode identifications are in agreement with the range of modes visible in disk integrated light of an equator-on visible pulsating component.

The wavelength distribution of pulsation amplitudes in AS Eri is largest in the Strömgren $u$ filter and decreases toward longer wavelengths. We place AS Eri and other known mass-accreting pulsating components of Algols on HR-diagram. They are located inside the instability strip on the Main Sequence. We also discuss the peculiar evolutionary status of primary components in Algols and stress that they are not normal $\delta$ Scuti stars, but form a separate group of pulsators. Finally, we discuss proximity and eclipse effects, and have simulated the effect of primary minimum data gaps that may produce the $1 / P_{\text {orb }}$ alias sidelobes in DFT analysis of eclipsing binary data. Aliases from gaps in primary minimum observations seem to be the principal limitation on spectral window functions in asteroseismic studies of eclipsing binaries.
\end{abstract}

Key words. stars: binaries: eclipsing - stars: variables: $\delta$ Scu - stars: oscillations - stars: individual: AS Eri

\section{Introduction}

The known pulsating components in semi-detached Algol-type eclipsing binary systems (EBS) form a small group with eight members (see Table 3). Starting evolution as normal MS stars in detached binary systems, these stars have undergone rapid

Send offprint requests to: D. E. Mkrtichian, e-mail: david@arcsec.sejong.ac.kr mass-accretion, when the originally more massive component filled its Roche lobe. As Algols still show evidence of slow mass transfer, their present primaries are not in thermal equilibrium. According to definition (Mkrtichian et al. 2002b), this new group of pulsators consists of pulsating mass-accreting Main Sequence stars of spectral type B-F in semi-detached Algol-type systems. Hereafter we use "oEA" (oscillating EA) for mass-accreting pulsating components in Algols, to stress 
their differences from normal $\delta$ Scuti-type components of detached eclipsing binaries, and to segregate them from the earlier General Catalogue of Variable Stars (GCVS) designation "EA/DSCT" which, by default, includes $\delta$ Sct-type pulsators in detached systems.

We stress that oEA designation includes pulsators in semidetached Algols with both EA- and EB-type light curves, since difference in these types have little physical significance (Giuricin et al. 1983b). We argue that these poorly-studied stars are potentially very attractive for asteroseismology as they permit asteroseismic estimation of accretion rates (Mkrtichian et al. 2002b), eclipse mode-identification, and accurate mass and radius determinations in binaries with radial velocities measured for both components. In turn, accurate parameters should help with pulsation spectra modeling.

The main goal of this work is to investigate the pulsation spectrum of AS Eri, and to search for evidence of pulsation-toorbital period resonances.

\section{AS Eri}

AS Eri $(V=8.31 \mathrm{mag})$ is a well known Algol-type semi-detached eclipsing binary with an orbital period of 2.664152 days. The A $3 \mathrm{~V}, 1.92 M_{\odot}$ and $1.80 R_{\odot}$ primary component is well within its Roche lobe. The K0 IV, $0.207 M_{\odot}$ and $2.25 R_{\odot}$ secondary subgiant fills its Roche lobe. The component absolute magnitudes are respectively $M_{\mathrm{v}}^{\mathrm{pr}}=1.77$ and $M_{\mathrm{v}}^{\mathrm{sec}}=4.18$ (Popper 1973). Van Hamme \& Wilson (1984) found improved parameters for the system and third light in the system. Their absolute parameters for the primary give an estimate of the primary's radius of $R=1.57 \pm 0.06$ that put it closer to the ZAMS radius. AS Eri evolved to the present configuration with substantial loss of angular momentum (Eggleton \& Kiseleva-Eggleton 2002). Struve \& Huang (1956) found evidence for emission from a gas ring around the primary, whose intensity varies with time. According to Koch (1960) out-of-eclipse parts of the light curve show significant seasonto-season variability that can be explained by variability of one component, or variable light introduced by a gas stream. Pulsations of the primary component of AS Eri with period $P=24.39 \mathrm{~min}$ and variable semi-amplitude were discovered by Gamarova et al. (2000) during the Central Asian Network's collaborative survey of pulsational variability among the components of eclipsing binaries (Mkrtichian et al. 2002a).

AS Eri was selected for a year 2000 multisite photometric campaign to examine additional excited modes of the primary A3 component.

\section{Observations}

During the pre-campaign run in January-February 2000 (JD 2451566, JD 2451572 and JD 2451580) AS Eri was observed photoelectrically at the Tien-Shan Astronomical Observatory (TSAO) of the Fesenkov Astrophysical Institute, Kazakhstan with a Johnson $B$-filter. These observations clearly revealed amplitude modulation of the pulsation and possible multiperiodicity. The multi-site campaign, started in September-December 2000, involved observatories in Spain,
Table 1. Log of 2000 year observations.

\begin{tabular}{|c|c|c|c|c|}
\hline Date & Start & End & Filter & Site \\
\hline \multicolumn{5}{|c|}{ HJD 2451 000.+ } \\
\hline $22 / 23.01 .2000$ & 566.048 & 566.1905 & $B$ & TSAO \\
\hline 28/29.01.2000 & 572.069 & 572.1913 & $B$ & TSAO \\
\hline 05/06.02.2000 & 580.0776 & 580.1674 & $B$ & TSAO \\
\hline $22 / 23.09 .2000$ & 810.6219 & 810.6915 & ubvy & SNO \\
\hline $23 / 24.09 .2000$ & 811.5785 & 811.7006 & $u b v y$ & SNO \\
\hline $24 / 25.09 .2000$ & 812.6186 & 812.7020 & $u b v y$ & SNO \\
\hline $26 / 27.09 .2000$ & 814.6303 & 814.6990 & $u b v y$ & SNO \\
\hline $27 / 28.09 .2000$ & 815.6346 & 815.7031 & $u b v y$ & SNO \\
\hline 03/04.10.2000 & 821.2839 & 821.3621 & V & SO \\
\hline $04 / 05.10 .2000$ & 822.2419 & 822.2709 & V & SO \\
\hline 05/06.10.2000 & 823.2230 & 823.3522 & V & SO \\
\hline 08/09.10.2000 & 826.2910 & 826.3671 & V & SO \\
\hline $16 / 17.10 .2000$ & 834.6144 & 834.6870 & ubvy & SNO \\
\hline $09 / 10.11 .2000$ & 858.2396 & 858.2686 & V & TSAO \\
\hline $18 / 19.11 .2000$ & 867.2346 & 867.3729 & V & TSAO \\
\hline $18 / 19.12 .2000$ & 897.1693 & 897.2044 & V & TSAO \\
\hline $19 / 20.12 .2000$ & 898.1163 & 898.2952 & V & TSAO \\
\hline
\end{tabular}

Uzbekistan, Kazakhstan and South Korea. The observatories cover $9 \mathrm{~h}$ of longitude over the Eurasian continent. Comparison stars $\mathrm{C} 1=\mathrm{HD} 22240$ (SAO 130587, $V=$ $7.51 \mathrm{mag}, \mathrm{A} 2)$ and $\mathrm{C} 2=\mathrm{HD} 21887$ (SAO 130544, $V=$ 7.18 mag, G5) were constant in brightness during the campaign. Simultaneous $u v b y$ photometry was collected at the Sierra Nevada Observatory (SNO), Spain, using the multichannel Strömgren-Crawford spectrograph photometer attached to the 0.90-m telescope (Rodriguez et al. 1997). Following these authors, we tranformed our $u v b y$ data to the standard Strömgren system. The resulting $y$ data are close to the standard Johnson $V$ system. Single channel $V$ observations were collected at the TSAO using the 0.48-m telescope with the "Odessa-TSAO" photometer. Observations at Sobaeksan Optical Astronomy Observatory (SOAO), South Korea, were collected using the PM512 CCD camera (field of view $4.3 \times 4.3$ arcmin) attached to the $0.61-\mathrm{m}$ reflector. These data were obtained and reduced using procedures described by Kim et al. (2002a). A Johnson $V$ filter and $15 \mathrm{~s}$ integrations were used. Because of poor weather, no observations were obtained at Maydanak Observatory (Uzbekistan) during the scheduled time.

From September to December 2000, observations were obtained on 14 nights, spread over 88 days. Two nights of marginal quality were omitted from the frequency analysis. A total of $28.3 \mathrm{~h}$ of usable photometry was obtained at three sites. The log of all 2000 observations is given in Table 1 .

\section{Time series analysis}

Differential magnitudes between AS Eri and SAO 130587 were used in the time series analysis. The original $V$ and $y$ magnitudes obtained during the campaign, and folded with the orbital period, are shown in Fig. 1. For extraction of pulsations we chose only out-of-eclipse parts of light curves and 
Table 2. Three frequency solutions for the 2000 multisite $V$-data for the Set 3.

\begin{tabular}{llcccc}
\hline \hline $\begin{array}{l}\text { Frequency } \\
\mathrm{d}^{-1}\end{array}$ & $\begin{array}{c}\sigma_{\mathrm{f}} \\
\mathrm{d}^{-1}\end{array}$ & $\begin{array}{c}\text { Semi-amplitude } \\
\mathrm{mag}\end{array}$ & $\begin{array}{c}\sigma_{\mathrm{A}} \\
\mathrm{mag}\end{array}$ & $\begin{array}{c}Q \\
\text { days }\end{array}$ & $\begin{array}{c}\text { Tentative identification } \\
l, m, n\end{array}$ \\
\hline$f_{1} \quad 59.03116$ & \pm .00005 & 0.0034 & \pm 0.0004 & 0.0120 & $1,1,5$ \\
$f_{2} \quad 62.5631$ & \pm .0001 & 0.0020 & \pm 0.0006 & 0.0113 & $2,-2,6$ \\
$f_{3} \quad 61.6743$ & \pm .0001 & 0.0013 & \pm 0.0005 & 0.0115 & $n=6$ \\
\hline
\end{tabular}

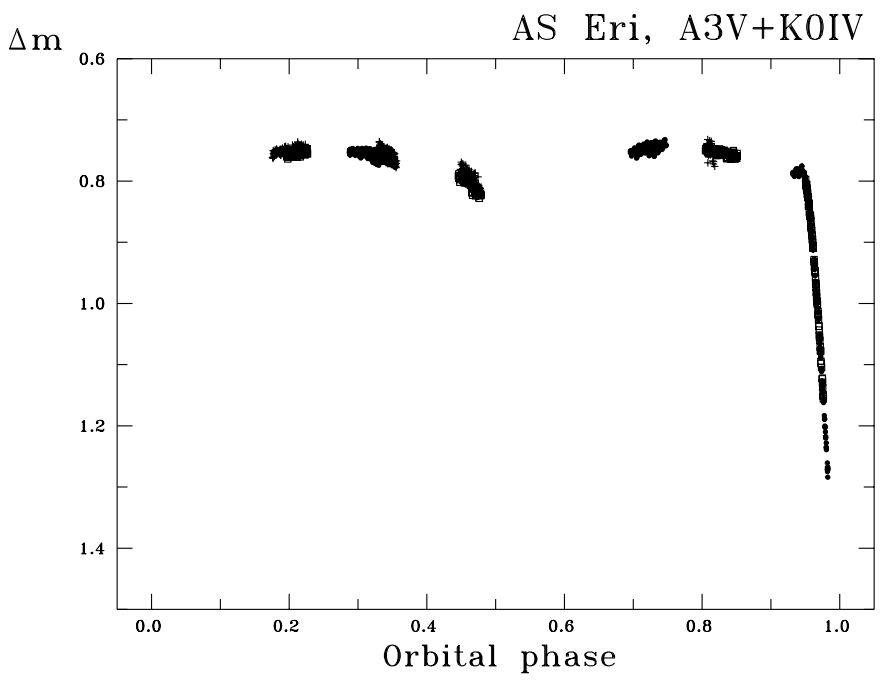

Fig. 1. Multisite campaign $V$ and $y$ differential light curve folded with the GCVS orbital ephemeris $\operatorname{JD}(\min )=2428538.066+$ $2.664152 P_{\text {orb }}$.

subtracted the trends produced by slow orbital light variations. Because of the near equality of pulsation amplitudes of A-F stars in Johnson $V$ and Strömgren $y$, we combined the SNO $y$ data with TSAO and SO $V$ data for further analysis. The extracted pulsational $B$ (for pre-campaign observations at Tien Shan Observatory) and the campaign's $V$ light curves are presented in Figs. 2 and 3, respectively. Different symbols denote observations at different sites (see legends). The time series analysis was performed using the Discrete Fourier Transform (DFT) program PERIOD (Breger 1990) and the program "Four-m" (Andronov 1994). The latter fits the multi-frequency signals simultaneously with the set of frequencies and harmonics, performing least-squares differential corrections. At each step of the frequency analysis we pre-whitened the data with all frequencies, using the optimized values of amplitudes and phases found in previous steps of the analysis. The September-December 2000 campaign (JD $2451810-2451898$ ) data have complex spectral window patterns with strong $\pm 1,2,3 \ldots \mathrm{d}^{-1}$ aliases giving rise to the corresponding frequency uncertainty. To minimize this uncertainty in frequency peak selection we proceeded with sets of data having different spectral window functions as follows. First, we did the analysis of the main set of campaign $V$ and $y$ nights that span the JD $2451810-2451898$ range (Set 1). Second, we analyzed only the sub-set of Tien-Shan and Sierra Observatory data (Set 2) having the best photometric quality. Third, we decided to include in the analysis as many nights of good quality as possible, and merged Set 2 with the pre-campaign JD 2451566-2451580 B filter data set scaled to the $V$ filter one using the scaling coefficient 1.32 . The resulting Set 3 spans 332 days. The frequency analysis of Sets 1, 2 and 3 led to similar results for the first three frequencies. Set 3 gives more accurate frequency estimates. We shall discuss below only the analysis for the best quality Sets 2 and 3. The DFT amplitude spectra obtained during the successive pre-whitening procedure are shown respectively in Figs. $4 \mathrm{a}$ and $4 \mathrm{~b}$ from top to bottom panels. The spectral window function of the Set 2 is shown in the top panel of Fig. 6. Frequency $f_{1}=59.03116 \pm 0.00005$ $\left(P_{1}=24.39390 \pm 0.00002 \mathrm{~min}, A_{1}=0.0034 \pm 0.0001 \mathrm{mag}\right)$ was found in the first analysis, in good agreement with the value $59.03 \mathrm{~d}^{-1}$ found in Gamarova et al. (2000).

The amplitude spectra of residuals (see middle panels of Figs. $4 \mathrm{a}$ and $4 \mathrm{~b}$ ), after subtracting the dominant oscillation $f_{1}$, shows the peaks resulting from the spectral window function and the probable multi-frequency low-amplitude signal. Two highest residual peaks of Set 2 and Set 3 are at frequencies $62.56 \mathrm{~d}^{-1}$ and $63.56 \mathrm{~d}^{-1}$, with amplitudes 0.00174 and $0.00179 \mathrm{mag}$ (Set2) respectively, and 0.00165 and $0.00155 \mathrm{mag}$ (Set 2). The $63.56 \mathrm{~d}^{-1}$ peak of Set 2 is an $1 \mathrm{~d}^{-1}$ alias of $f_{2}=62.56 \mathrm{~d}^{-1}\left(P_{2}=23.01 \mathrm{~min}, A_{2}=0.0017 \mathrm{mag}\right)$. The $62.5631 \mathrm{~d}^{-1}$ has larger amplitudes in Set 3 and Set 1 , and gives a better approximation of residual data of both sets.

The statistical significance of the $f_{2}=62.5631 \mathrm{~d}^{-1}$ peak was estimated using the bootstrap randomization technique (Murdoch et al. 1993; Kürster et al. 1997). The measured photometric magnitude values were randomly shuffled keeping the observed times fixed. A Lomb-Scargle periodogram was then computed for each "random" data set. The fraction of a large number of periodograms (200000 in this case) having power higher than the $f_{2}$ peak in the range $50<f<70 \mathrm{~d}^{-1}$ represents the false alarm probability (FAP) that noise would create the detected signal. In the Set 3 data DFT the FAP for $f_{2}=62.5631 \mathrm{~d}^{-1}$ peak peak was $<5 \times 10^{-6}$. The bottom panels in Figs. 4a,b show the DFT amplitude spectrum of residuals in Set 2 and Set 3 after subtracting the $f_{1}$ and $f_{2}$ frequencies with optimized amplitudes and phases.

The third, statistically significant signal at $f_{3}=$ $61.6743 \mathrm{~d}^{-1}$ is undoubtedly present in the pulsations of star; this peak found in DFT of both data sets has semi-amplitude $A=0.00125 \mathrm{mag}$ and the FAP $<5 \times 10^{-6}$ in the Set 3 . However its exact frequency found in DFT must be considered as preliminary due to uncertainty in the spectral window function. In Table 2 we give the parameters of least-squares fit for the longest Set 3. 


\section{$\Delta B(m a g)$}

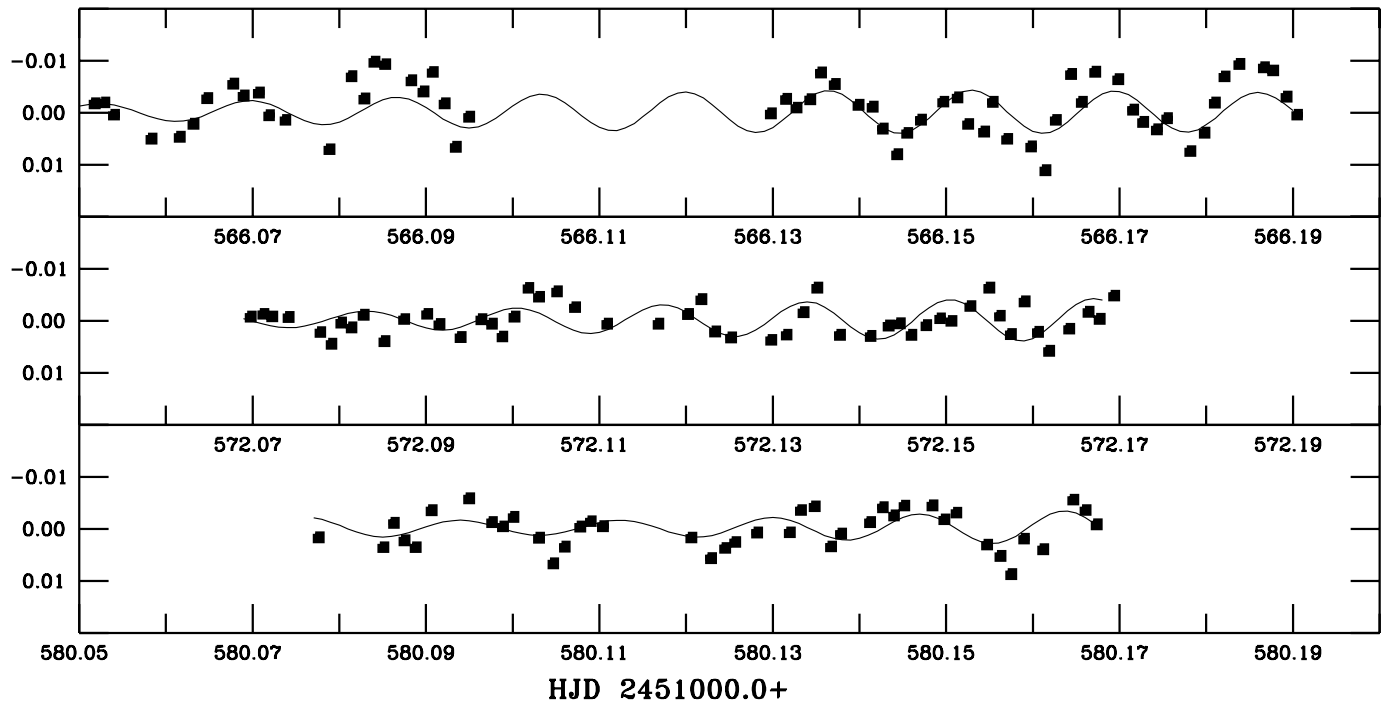

Fig. 2. Pulsational $V$ light curves of AS Eri pre-campaign observations at the Tien-Shan Observatory. Binary light variations are subtracted.

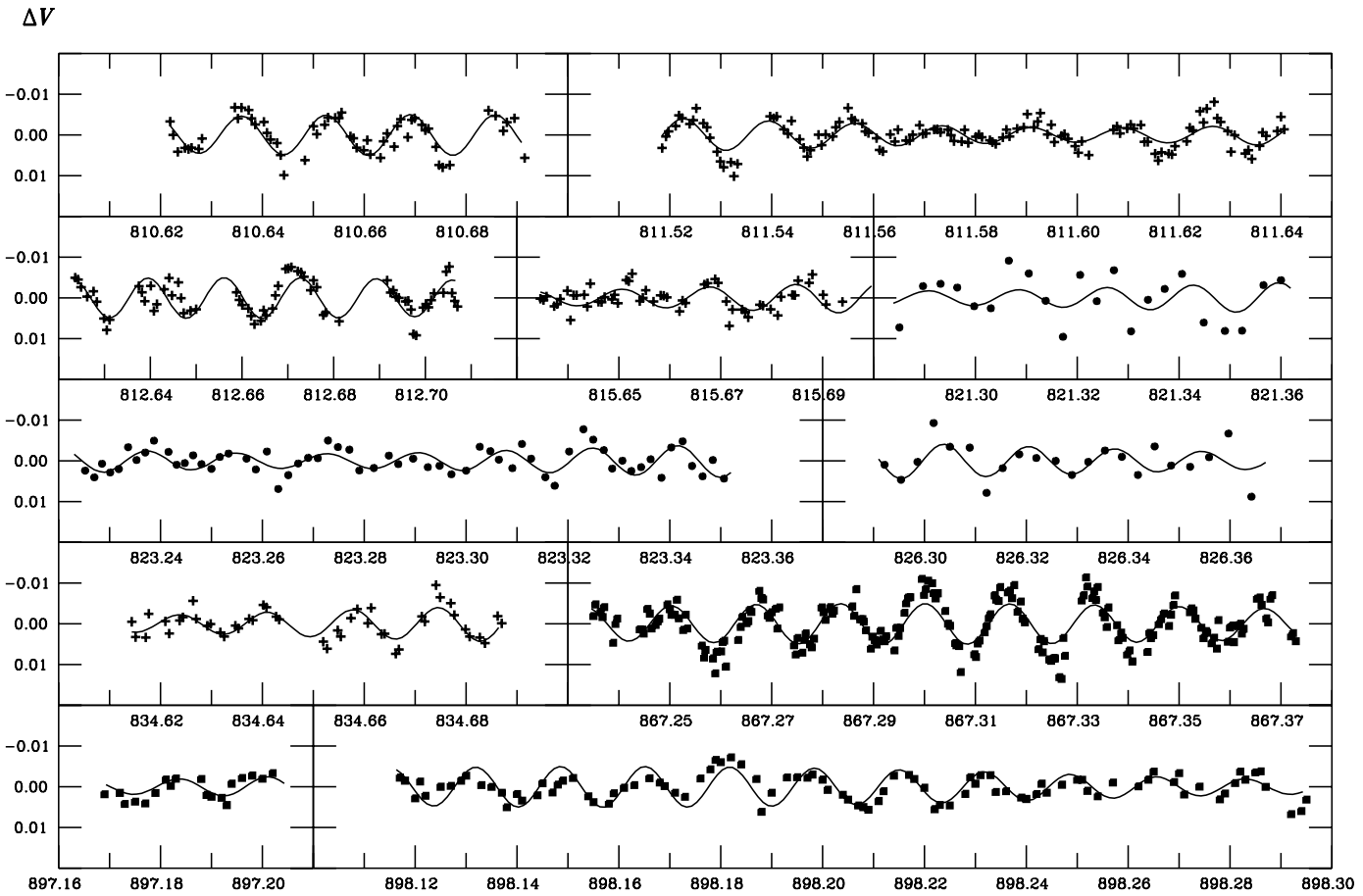

Fig. 3. Pulsational $V$ light curves of AS Eri: crosses - SNO, dots - SOAO, squares TSAO. Binary light variations are subtracted.

\section{Wavelength dependence of pulsation amplitude and mode identifications}

To examine the wavelength dependence of pulsation amplitude we fitted the multicolor $u, v, b, y$ subset of SNO data in each filter with the precise pulsation frequencies $f_{1}$ and $f_{2}$.

The following pulsation amplitude ratios $K_{1}^{u / v}=1.41$, $K_{1}^{v / y}=1.51, K_{1}^{b / y}=1.33$ and $K_{2}^{u / v}=1.59, K_{2}^{v / y}=1.85$, $K_{2}^{b / y}=1.73$ were found in AS Eri respectively for $f_{1}$ and $f_{2}$ high $S / N$ ratio modes.
As seen, the maximum amplitude is in the $u$ filter. It decreases monotonically towards longer wavelengths. Such a distribution in the UV is not typical of $\delta$ Sct stars, but rather of hotter pulsating stars such as $\beta$ Cep (Heynderickx et al. 1994) or slowly-pulsating B stars (Waelkens 1996). Some $\delta$ Scuti stars located near the blue border of the instability strip (for instance HD 129231) show such a feature (Rodriguez et al. 2001). Rodriguez et al. (2004) show that for normal $\delta$ Sct-type stars the higher $u$ amplitudes are due to the effect of highovertone pulsations and small pulsation constant values. 


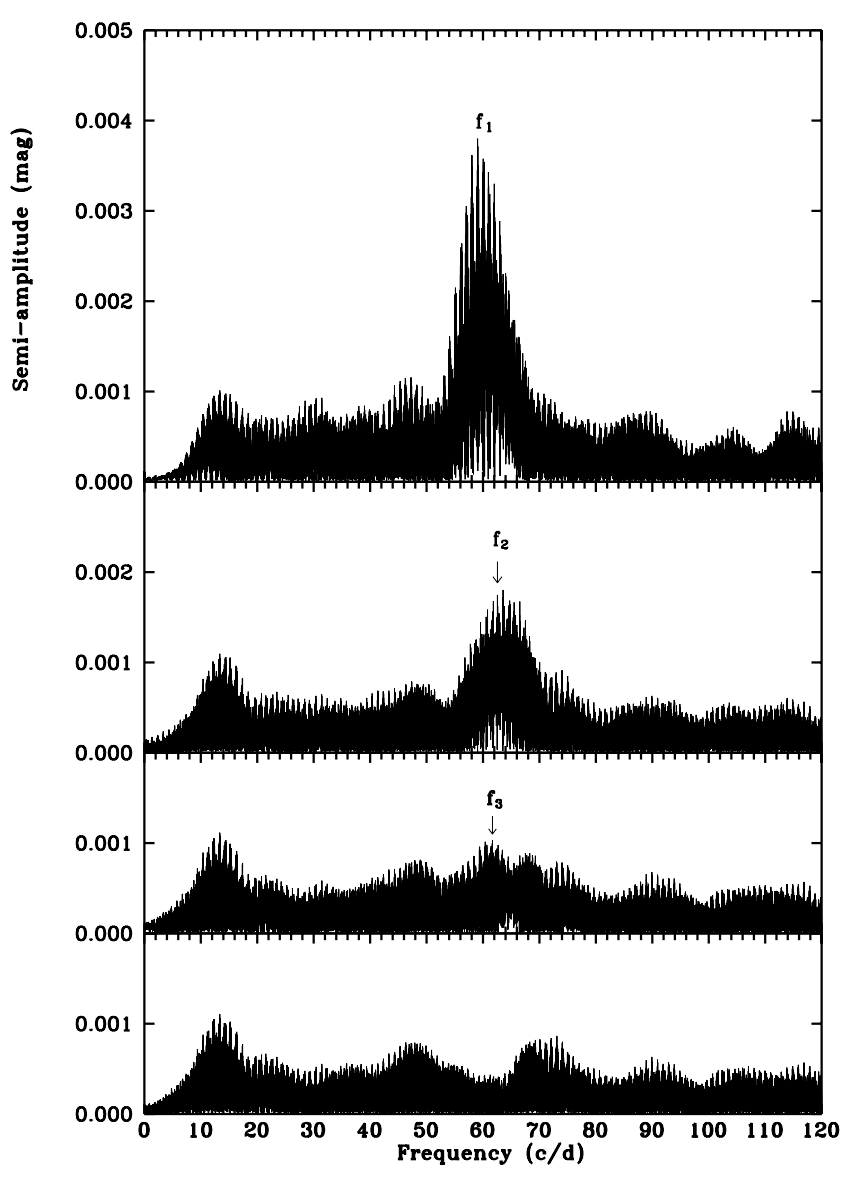

a)

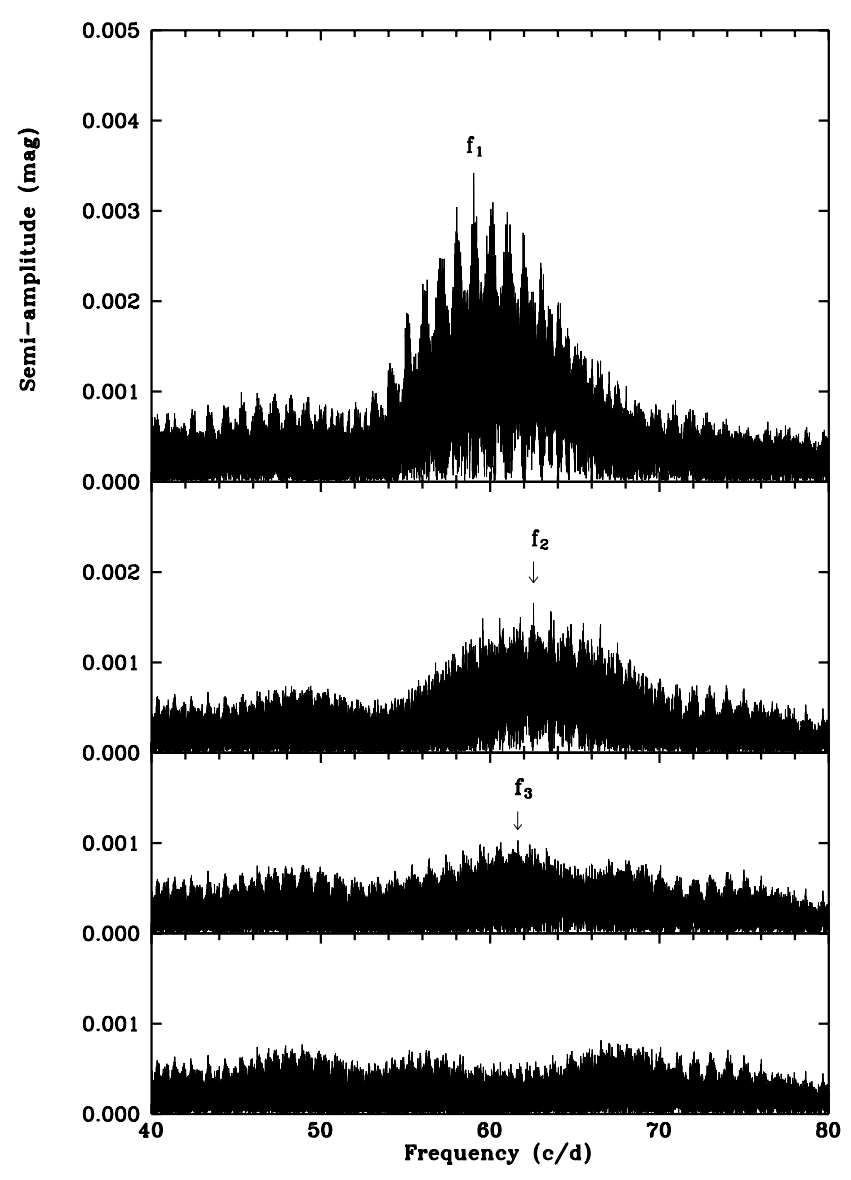

b)

Fig. 4. Amplitude spectra of frequency analysis of the Set 2 a) and Set $3 \mathbf{b}$ ). The steps of the pre-whitening procedure are shown from top to bottom.

On the other hand, such a peculiarity is not surprising for AS Eri, if we recall that eclipsing oEA stars are accreting mass mainly at equatorial latitudes. For direct stream-star impact systems, such as AS Eri, the infalling hypersonic gas stream penetrates the atmospheric layers to the point where the gas pressure equals the ram pressure of the stream, and produces shock waves. Gas-stream kinetic energy is thereby converted to thermal energy. For the $\dot{M}>3 \times 10^{-9}$ the shock appears below the photosphere, drifting outwards toward the photosphere with decreasing accretion rates (Ulrich \& Burger 1976). Therefore the equatorial latitudes of the primary component in AS Eri in the zone of impact and accretion may be heated by UV radiation. Due to the asynchronous rotation of AS Eri (see Sect. 6.2), this hot equatorial zone can extend in longitude. The hot surface zone may shift the maximum pulsation amplitude to the UV band, unlike normal non-accreting main sequence stars of the same spectral class. On the other hand, excited sectoral modes (see results of mode identification for AS Eri in Sect. 6.2) have maximum surface amplitudes on equatorial latitudes, where accretion occurs. The pulsation amplitude distribution versus wavelength for these modes may have a peculiar character, and may help identify the spatial structure of the observed modes. This hypothesis could be checked by analysis of the amplitude modulation in different wavelength bands over the orbital period. Unfortunately, due to large error bars in phase determinations for available multicolor data, the application of Watson's technique (Watson 1988; Garrido et al. 1990) for reliable tentative mode identifications cannot be performed.

However, we can narrow the range of possible identifications for excited modes based on two facts: i) the eclipsing binaries are (according to the definition of "eclipsing") observationally pre-selected binaries with a orbital plane that lies close to line-of-sight and the pulsating components are (if we assume that rotation axes of the components are perpendicular to the orbital plane) equator-on visible stars ii) the disk-integrated photometric amplitudes decrease drastically for modes with $l>2$. For near equator-on-visiblity with inclination $i=82.98^{\circ}$ (Van Hamme \& Wilson 1984) AS Eri disk-integrated mode amplitudes having $(l+|m|)=$ odd tend to be close to zero. This means that only radial and $l, m=(1, \pm 1), l, m=(2, \pm 2)$, $l, m=(2,0)$ non-radial modes survive in the signal integrated over the stellar disk.

The accurate absolute parameters of the AS Eri system make possible the identification of the radial order $(n)$ for nonradial modes by comparing theoretical and calculated pulsation constants

$Q=P\left(\rho / \rho_{\odot}\right)^{0.5}$ 
Table 3. The group of pulsating mass-accreting primary components of semi-detached Algol-type eclipsing binaries. Absolute parameters are given for primary components and references are given for pulsation periods.

\begin{tabular}{lllllllll}
\hline \hline Name & \multicolumn{1}{c}{$\begin{array}{c}\mathrm{Sp} \\
\text { (Prim. + Sec.) }\end{array}$} & \multicolumn{2}{c}{$M / M_{\odot}$} & $\begin{array}{c}R / R_{\odot} \\
\text { (Primary) }\end{array}$ & $\begin{array}{l}\log T_{\mathrm{e}} \\
\log L / L_{\odot}\end{array}$ & $\begin{array}{l}P_{\text {orb }} \\
\text { (days) }\end{array}$ & $\begin{array}{l}P_{\text {puls }} \\
\text { (min) }\end{array}$ & References \\
\hline Y Cam & A7 V + K1 IV & - & - & 3.868 & - & 3.3055 & $95.70,78.75$, & Kim et al. (2002a) \\
& & & & & & & $97.16,81.2$ & \\
AB Cas & A3 V + K0 IV & 2.3 & 1.97 & 3.903 & 1.28 & 1.3669 & 83.93 & Rodriguez et al. (1998) \\
RZ Cas & A3 V + K0 IV & 2.205 & 1.67 & 3.934 & 1.12 & 1.1953 & $22.43,25.4$ & Lehmann \& Mkrtichian (2002) \\
R CMa & F1 V + G2-K2 IV & 1.07 & 1.48 & 3.864 & 0.76 & 1.1359 & 68.5 & Mkrtichian \& Gamarova (2000) \\
TW Dra & A6 V + K0 IV & 1.7 & 2.4 & 3.922 & 1.4 & 2.8069 & 80 & Kusakin et al. (2001) \\
AS Eri & A3 V + K0 III & 1.93 & 1.57 & 3.928 & 1.06 & 2.6642 & $24.39,23.01$, & Present paper \\
& & & & & & & 23.34 & Kim et al. (2002b) \\
RX Hya & A8 V + K5 IV & - & - & - & - & 2.2816 & 74.26 & Kim et al. (2002c) \\
AB Per & A5 V + G9 IV & - & - & - & - & 7.1601 & 282.0 & \\
\hline
\end{tabular}

where $P$ is pulsation period and $\rho$ and $\rho_{\odot}$ are the mean densities of the star and the Sun, respectively. Adopting the improved (probably more accurate) estimates of the absolute parameters of the primary $M=1.93 M_{\odot}$ and radius $R=1.57 R_{\odot}$ from Van Hamme \& Wilson $(1984,1990)$ the mean density of the primary is $\rho / \rho_{\odot}=0.4987$. The pulsation constants are for 59.03116, 62.5631 and $61.6743 \mathrm{~d}^{-1}$ oscillations respectively as $Q_{1}=0.0120, Q_{2}=0.0113$ and $Q_{3}=0.0115$. The comparison of these values with the theoretical one (Fitch 1981) for $l=2$ modes relate them respectively to the fifth $\left(f_{1}\right)$ and sixth ( $f_{2}$ and $f_{3}$ ) overtone pulsations. Note that these $P_{1}=24.39$, $P_{2}=23.01$ and $P_{3}=23.34$ min oscillations in AS Eri and the 22.4 min oscillations found recently in the A3 component of the Algol-type system RZ Cas (Ohshima et al. 1998, 2001) are the shortest known periods excited in the non-degenerate, non-magnetic main sequence A-type stars.

\section{Discussion}

\subsection{Location in the instability strip}

The purpose of this subsection is to discuss further peculiarities of oEA stars relative to classical $\delta$ Scuti stars, to show that they form a separate pulsator group and to find their positions in HRdiagram. Figure 5 shows the location of the A3 V component of AS Eri, and other oEA stars with accurately known absolute parameters on the $\log T_{\mathrm{e}}-\log L$ diagram. Absolute parameters for these systems were adopted from Popper (1973), Mezzetti et al. (1980), Budding (1985), Maxted et al. (1994), Sarma et al. (1996), Ando (1980), Giuricin et al. (1983a) the ZAMS and the TAMS parameters are taken from evolutionary calculations of De Greve (1993). As can be seen, AS Eri and other pulsating gainers lie inside the classical $\delta$ Scuti star instability strip close to the ZAMS. Table 3 summarizes the main parameters of oEA stars. Tentative values of pulsation frequencies are given in the seventh column. The primary components in Algols and the pulsating component in AS Eri are MS stars of a special type. The majority of oEA stars were formed as low-mass companions in detached binary systems. Evolution of the more massive components led to Roche lobe overflow (RLOF) and rapid mass transfer (RMT) onto the lower mass components. During Case B evolution (Paczynski 1971), a significant part of the transferred mass was captured by the present primary pulsating components. During this RMT stage of "negative" evolution, mass-gainers moved to higher mass and luminosity, finally settling (at the onset of the slow mass-transfer (SMT) stage) on the HR-diagram closer to the ZAMS. Accretion of mass on the thermal time scale rejuvenates the gainers, increasing their central hydrogen content by convective mixing (De Greve 1993). The separation of gainers from the ZAMS reflects the central hydrogen content (De Greve et al. 1985).

As an example, the typical evolutionary track (including MS, RMT and SMT stages) of a gainer of initial mass $1.8 M_{\odot}$ in the $3.0 M_{\odot}+1.8 M_{\odot}$ and initial $P_{\text {orb }}=2.5$ day binary system evolving through the case B mass transfer taken from De Greeve's (1993) evolutionary sequences database is shown by the dotted line in Fig. 5. Evolutionary tracks, physical parameters at the end of RMT and onset of slow masstransfer stages and degree of rejuvenation depend on initial masses and mass ratios, orbital periods, angular momentum, mass-accretion and mass-loss rates from the system during its evolution (see for instance De Greve 1993; Nelson \& Eggleton 2001).

In spite of the fact that oEA stars occupy the lower instability strip and show pulsational characteristics inherent to $\delta$ Sct stars, they cannot be considered ordinary $\delta$ Sct stars because of their previous evolutionary life. oEA stars also do not follow the evolutionary tracks of normal MS or post MS $\delta$ Sct stars. Being at present in a semi-detached configuration and in the SMT stage, oEA stars are still accreting mass at rates ranging (for different systems) between $10^{-6}-10^{-11} \dot{M}_{\odot}$ per year. Slow, and possibly non-stationary, accretion keeps them in thermal inbalance and causes the slow evolution along the $M S$ toward higher mass and earlier spectral type. For this reason oscillating oEA stars are attractive for asteroseismology.

\subsection{Synchronization and resonance of orbital and pulsation periods}

The hypothesis of high order pulsation-to-orbital period synchronization by tidal resonance is naively very attractive, because of the many resonances seen in nature and in stellar and 


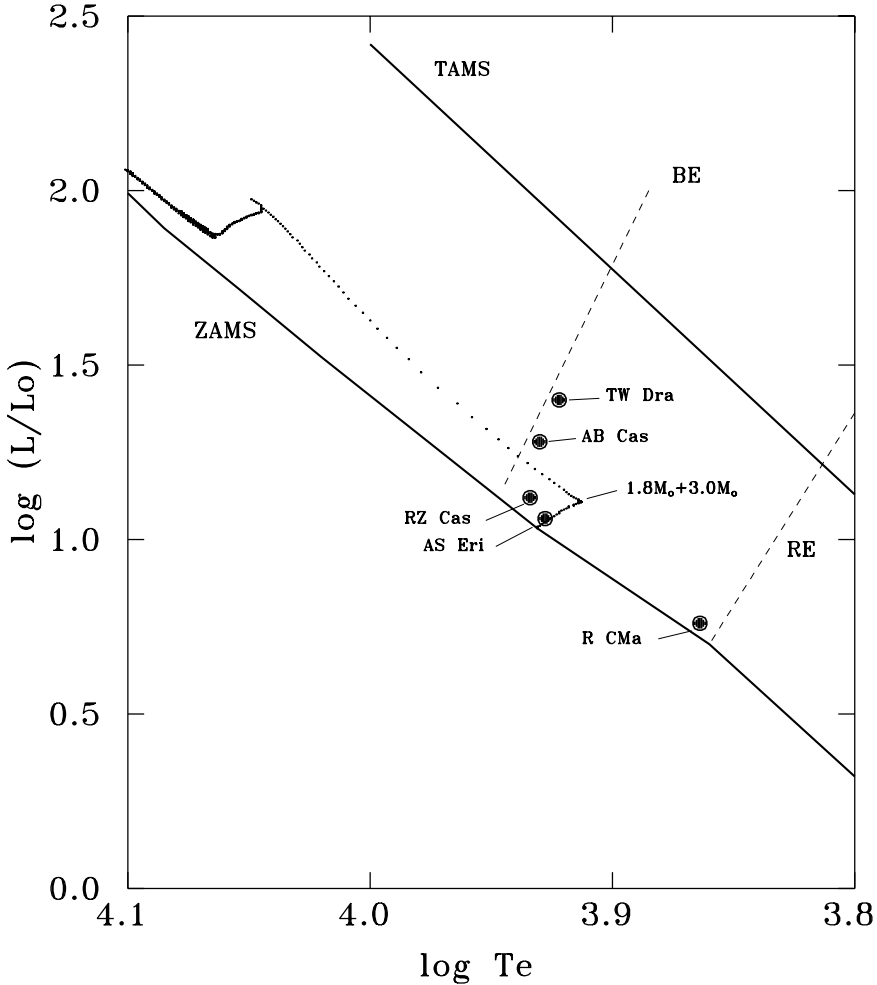

Fig. 5. Positions of oEA stars with accurately known absolute parameters on $\log T_{\mathrm{e}}-\log L / L_{\odot}$. The blue (BE) and red (RE) edges of the instability are shown by dashed lines. The dotted line shows the evolutionary track of a $1.8 M_{\odot}$ gainer in a $3.0 M_{\odot}+1.8 M_{\odot}$ binary system evolving through Case B (De Greve 1993).

planetary environments. While attractive, the theoretical basis of probable high-order resonances for acoustic modes has not yet been developed and understood and the resonances are not well confirmed observationally. On the other hand, if it exists, pulsation-to-orbital synchronization (POS) might be significant for understanding the nature of such resonances.

Observational evidence for high pulsational stability in binary systems was suspected, and the hypothesis of direct synchronization of pulsation orbital periods in some Algol systems was suggested by Frolov et al. (1980). Recently, this hypothesis was checked but not confirmed for the semi-detached eclipsing binary AB Cas (Rodriguez et al. 1998).

Strong evidence of the low-order POS in a eclipsing binary in the open cluster NGC 2126 was found by Gáspar et al. (2003). The authors discovered that V6 is an eclipsing binary with orbital period $P_{\text {orb }}=1.17320$ and has a pulsating component with a period of 0.12936 days. That gives the $P_{\text {orb }} / P_{\text {puls }}=9.07$ ratio - that may indicate a POS. The question presence of the resonanse is still unanswered, and should be checked with accurate pulsation and orbital periods of a larger sample of detached and Algol-type eclipsing binary systems. Below, we attempt to check this hypothesis and the exact commensurability of pulsation and orbital periods for AS Eri.

We stress that use of apparent observed pulsation periods to seek the orbital-to-pulsation period ratios, and to check the direct synchronization hypothesis, is valid only for radial $(l=0)$ and zonal $(m=0)$ modes. For nonradial modes with $m \neq 0$

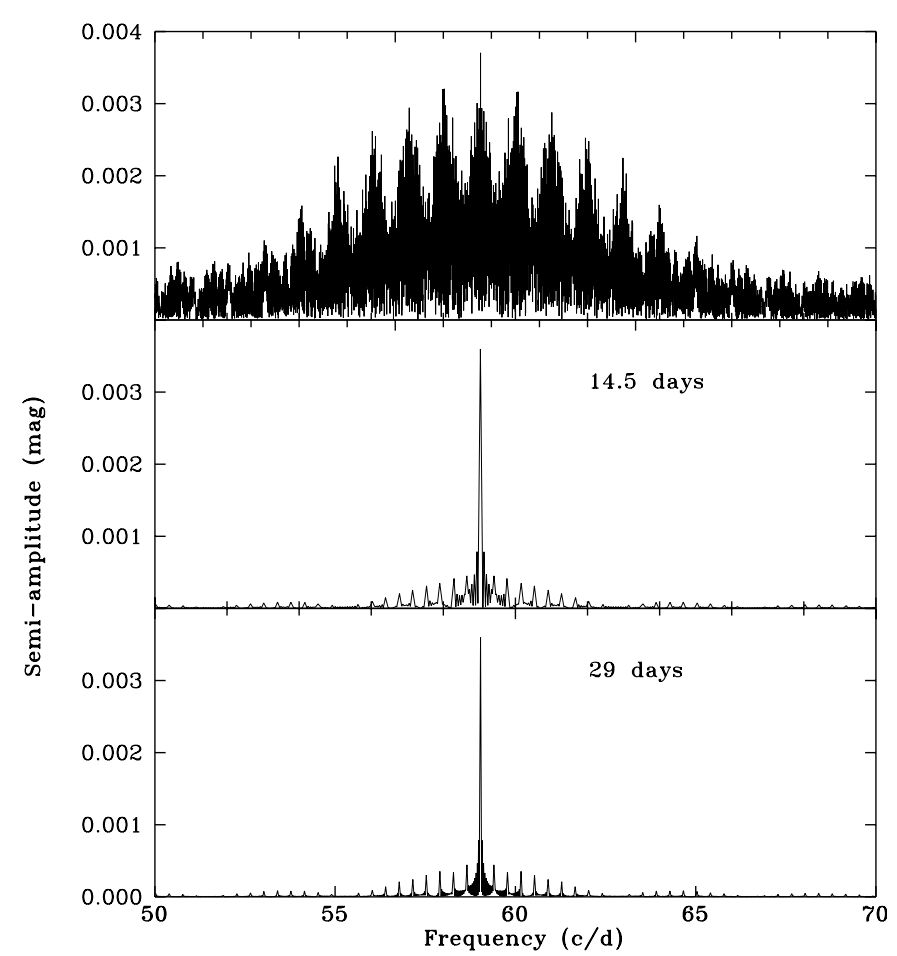

Fig. 6. (Top panel) the spectral window functions for the AS Eri campaign Set 2 data point distribution; (middle panel) for a simulation of continuous 14.5-day space-based observations; (bottom panel) for 29 days of photometric observations (with a sampling 0.001 days) with primary minima gap duration $0.2 P_{\text {orb }}$. The $f_{1}=59.0311 \mathrm{~d}^{-1}$ frequency, semi-amplitude 0.0036 mag for simulated data were used.

azimuthal numbers, the rotational Doppler effect on pulsation frequencies should be included to obtain the true oscillation frequencies in the frame corotating with the pulsating component. For rigid rotation, the first order frequency correction term for the Doppler effect is (Cox 1984)

$f_{\text {cor }}^{l, m, n}=f_{\text {obs }}^{l, m, n}+m f_{\text {rot }}$

where $f_{\text {cor }}^{l, m, n}, f_{\text {obs }}^{l, m, n}$ and $f_{\text {rot }}$ are respectively the frequency of the mode in a corotating system, the observed frequency and the rotation frequency; $l, m, n$ are respectively the degree, the azimuthal and the radial quantum numbers describing the spatial structure of nonradial modes.

Hence, to find the pulsation-to-orbital period ratio coefficient, we require not only precise apparent oscillation and rotation frequencies but also the azimuthal quantum number $m$ of the observed mode. For the case of $K=9.0$ POS for the observed period of the pulsating component of binary V6 in NGC 2126 we suggest radial mode identification for the dominant oscillation.

For exact spin-orbital synchronized systems $\left(F_{\text {asyn }}=\right.$ $f_{\text {rot }} / f_{\text {orb }}=1$ ), a formal check of the direct POS hypothesis (but not the correct period ratio) is possible having accurate values of apparent oscillation frequencies. The unknown $m= \pm 1,2 \ldots$ numbers in the frequency correction terms in Eq. (2) results in adding or subtracting the corresponding integer numbers $m= \pm 1,2$. . to period ratios obtained from apparent oscillation frequencies. This is not valid for AS Eri, known to be an asynchronous system (Giuricin et al. 1984). 
We also stress that $F_{\text {asyn }}=V_{\text {obs }} / V_{\text {syn }}$ (observed to synchronous rotational velocity ratios) determined in Algols spectroscopically from $v \sin i$ values might be affected by variable circumstellar envelope or accretion bulge absorption effects or accretion-driven differential rotation of the equatorial atmopsheric layers, and may be inaccurate. Moreover, for some well-studied Algols (perhaps because of these effects), $v \sin i$ values appear to vary from line to line and occasionally with time (see discussion and the references in Etzel \& Olson 1993). Therefore, for many Algols, spectroscopically determined spin-orbital asynchronicity coefficients are not accurate enough to use in asteroseismic applications. That is, such coefficients do not yield sufficiently accurate pulsation frequencies in the frame co-rotating with the pulsating star.

With this in mind, and having the tentative range of $(l, m)$ mode identifications found in the previous section, we attempted to check formally the direct POS, assuming consecutively the trial radial and $m=0, \pm 2, \pm 1$ identification for the $f_{1}$ and $f_{2}$ modes. We adopt the only available estimate of mean rotational velocity $V_{\text {obs }} \simeq 35 \mathrm{~km} \mathrm{~s}^{-1}$ given by Popper (1973) and estimate of the synchronous velocity $V_{\text {syn }}=29.8 \mathrm{~km} \mathrm{~s}^{-1}$ using the primary radius $1.57 R_{\odot}$ (Van Hamme \& Wilson 1984). This leads to a rough estimate of the primary star spin-orbital asynchronicity coefficient of $F_{\text {asyn }}=f_{\text {rot }} / f_{\text {orb }}=V_{\text {obs }} / V_{\text {syn }}=1.17$. The rough estimate of the rotation frequency of the primary is $f_{\text {rot }}=f_{\text {orb }} \cdot F_{\text {asyn }}=0.441 \mathrm{~d}^{-1}$.

Following Eq. (2) we calculated the expected frequencies of $f_{1}$ and $f_{2}$ in the corotating frame and pulsation-to-orbital frequency ratios obtained with the assumed trial values of the azimuthal quantum numbers $m=0, \pm 1, \pm 2$. We limit ourselves to degree $l \leq 2$, because disk integrated photometric amplitudes of $l \geq 3$ modes decrease drastically.

Only the frequency ratios $K_{1}=156.09$ for $m=1$ identification or the ratio $K_{1}^{\prime}=154.92$ for $m=2$ identification for the $f_{1}$ mode and $K_{2}=169.03$ and $m=-2$ identification for the $f_{2}$ mode are close to integer values and in formal agreement with POS. In other words, if the hypothetical pulsationalto-orbital period high-order synchronization occurs in AS Eri for $f_{1}$ and $f_{2}$ modes, then their azimuthal quantum numbers could be formally identified as $m=1$ or $m=2$ and $m=-2$ respectively. We stress that coincidence of frequency ratios with integer values may be accidental, the ratios are sensitive to the incertainties in rotation velocity determinations and this formal identification may be incorrect. On another hand, these $l, m=1,1$ or $l, m=2,2$ and $l, m=2,-2$ probable identifications from frequency ratios for $f_{1}$ and $f_{2}$ modes respectively, do not contradict to the range of mode identifications found in Sect. 5 that are based on equator-on-visibility of components of eclipsing binaries. This agreement suggests that these mode identifications found from POS assumption may be correct.

Finally, in spite of formal near-integer period ratios for trial mode identifications and formal evidence of POS it is premature to draw a firm conclusion about the direct pulsation-toorbital period synchronicity in AS Eri. The question about POS in AS Eri will remain open until independent photometric or spectroscopic confimations of mode identification have available, for instance, the application of primary eclipse modeidentification technique (Gamarova et al. 2003) to AS Eri.
Whether pulsational-to-tidal synchronisation and spin-orbital asynchronisation widely present in Algols should be checked observationally. Such investigations should begin with the detection of pulsations in a large sample of Algols. Later work should separate the effects of non-stationary accretion-driven variability from pulsations, and include detection of the complete spectrum and precise frequencies of excited modes, accurate mode identification, and asteroseismic determination of the rotational frequency of the pulsating components.

\subsection{The effects of binarity and eclipses}

This work is one of the first devoted to multisite studies of oEA stars. For completeness, we now discuss additional specific effects, limitations and artifacts that may arise in studies of pulsations in eclipsing binaries.

\subsubsection{The secondary component contribution and reflection effects}

The secondary component contribution to the total systemic brightness outside eclipses decreases the apparent pulsation amplitude of the primary star. Adopting the $V$ filter primary luminosity in units of total brightness of the system $L_{\mathrm{pr}} /\left(L_{\mathrm{pr}}+\right.$ $\left.L_{\mathrm{sec}}\right)_{y}=0.829$ (Van Hamme \& Wilson 1984) and using the equation for magnitudes

$\delta m=-1.086 \frac{\delta L_{\mathrm{pr}}}{L_{\mathrm{pr}}+L_{\mathrm{sec}}}$

we find the coefficient $K=1.206$ for transformation of the apparent amplitudes (given in the third column of Table 2) into real disk-integrated $V$ filter mode amplitudes in the pulsating component of AS Eri.

The variable secondary contribution leads to an increase of the apparent pulsation amplitudes in secondary minima, when the non-pulsating component is (partially or totally) eclipsed, and should be also be corrected (if pulsation light curves from these phases are included in the analysis), to avoid false periodicities in DFT spectra. The variable reflection effect in binaries, if not accounted for during extraction of the pure pulsation variability, may lead to false periodicity with $P_{\text {orb }}$ giving rise to a $1 / P_{\text {orb }}$ frequency splittings in pulsation spectra.

This effect should be corrected in the asteroseismology of EBS studying detection and mode identification of very lowamplitude modes. These false, small amplitude $1 / P_{\text {orb }}$ splitting of frequency peaks may be misidentified as the rotational mode splitting phenomenon. The reflection effect in AS Eri is not taken into account here in out-of-eclipse curves because of the small changes in relative magnitudes through the orbital phases covered in this campaign. Such changes introduce negligible effects on pulsation amplitudes, even for the highest-amplitude (3.7 mmag) $f_{1}$ mode.

\subsubsection{Effects of primary eclipse gaps}

Observations of pulsations of primary components in EBS are interrupted (for total eclipses) or screened (for partial eclipses) 
during the primary minima. These phases are very informative for mode identification because of specific pulsation amplitude and phase variability differently for different non-radial modes (see Nather \& Robinson 1974; Gamarova et al. 2003).

Those parts of binary light curves where pulsations are difficult to extract, and during which there may be strong phase and amplitude variability are usually omitted for data homogeneity in plain DFT analysis. This procedure was followed in the present paper. Omitting primary minima produces gaps in observations separated on $P_{\text {orb }}$. In DFT spectra these gaps result in $1 / P_{0}=f_{\text {orb }}$ aliases and complicate the search for NRP rotational mode splitting spaced approximately as $\pm m f_{\text {orb }}$ in the case where the pulsating component rotates synchronously with orbital period (i.e. $f_{\text {rot }}=f_{\text {orb }}$ ). To estimate the significance of this effect, we simulate continuous artificial data in 14.5 and 29 day-long spacecraft observations of AS Eri. These data were generated using the pulsational parameters for the $f_{1}$ mode, $0.2 P_{\text {orb }}$ duration of a gap around the primary minima and a data point step of 0.001 days. No random noise was included. Figure 6 shows the corresponding spectral window functions for 2000 year 88 day duration (Set 2) data (top panel), 14.5 days (middle panel) and 29 day (bottom panel) duration artificial spacecraft data. As can be seen, primary minima $0.2 P_{\text {orb }}$ gaps in the artificial data produce $1 / P_{\text {orb }}$ sidelobes having a sidelobe amplitude of about $12 \%$ of the main peak. The maximum sidelobe amplitude is independent of the time span of observation. This fact may be the principal limitation on spectral window functions of space and ground-based asteroseismic observations of eclipsing binaries. Here we ignore light-time effects of the order of several seconds for shortperiod Algols, which have little effect on pulsation phase.

\section{Conclusions}

In this study of rapid pulsations in the primary component of the Algol-type eclipsing binary system AS Eri, we find three excited intermediate overtone oscillation modes. Using the fact that orbital plane of the eclipsing binaries lie close to the lineof-sight we narrow the probable range of mode identifications and stress that only the modes with $(l+|m|)=$ even will be visible in disk integrated light. We discussed the probable existence of high-order pulsation-to-orbital period synchronization and checked period ratios under different assumptions about the trial mode identifications. Finally, we suggested the sectoral $(l, m, n=1,1,5$ or $2,2,5)$ and $(l, m, n=2,-2,6)$ mode identification of $f_{1}$ and $f_{2}$ modes respectively. We also find, for direct stream-star impact system such as AS Eri, that a typical pulsation amplitude distribution has a maximum in Strömgren $u$ band. Whether this is true for all pulsating oEA stars must be checked by analysis of the wavelength distribution of pulsation amplitudes in direct-impact pulsators and pulsators in systems with accretion disks. We need to improve the statistics on discovered oEA stars. We discussed the properties of presently known oEA stars and show that they form the separate group of pulsators inside the instability strip and have a different evolutionary status than $\delta$ Sct stars. oEA stars are evolving with the mass accretion along the Main Sequence. Finally, we discussed reflection and primary eclipse gap effects on the frequency spectrum and spectral window functions, and showed that gaps may produce $1 / P_{\text {orb }}$ alias sidelobes in DFT spectra of both ground-based and space EBS data. These aliases are the principal limitation in asteroseismic observations of eclipsing binaries when very low amplitude modes and rotational mode splitting are sought.

Acknowledgements. This work was the result of research activities of Astrophysical Research Center for the Structure and Evolution of the Cosmos (ARCSEC) supported by Korea Science \& Engineering Foundation and was also supported by Korea Research Foundation Grant (KRF-2001-015-DP0612). MDE is thankful to Dr. De Greve for providing the data base of his evolutionary calculations. KAV is grateful the Director of TSAO Dr. K. Kuratov for support for this and the CAN observing program at TSAO.

\section{References}

Ando, H. 1980, Ap\&SS, 71, 249

Andronov, I. L. 1994, Odessa Astron. Publ., 7, 49

Breger, M. 1990, Comm. Asteroseismol., 20, 1 (University of Vienna) Budding, E. 1985, PASP, 97, 584

Cox, J. P. 1984, PASP, 96, 577

De Greve, J. P. 1993, A\&AS, 97, 527

De Greve, J. P., \& Packet, W. 1989, A\&A, 230, 97

De Greve, J. P., de Landtsheer, A. C., \& Packet, W. 1985, A\&A, 142, 367

Eggleton, P. P., \& Kiseleva-Eggleton, L. 2002, ApJ, 575, 1037

Etzel, P. B., \& Olson, E. C. 1993, AJ, 106, 1200

Fitch, W. S. 1981, ApJ, 249, 218

Frolov, M. S., Pastukhova, E. N., \& Mironov, A. V. 1980, IBVS No. 2179

Gamarova, A. Yu., Mkrtichian, D. E., \& Kusakin, A. V. 2000, IBVS No. 4837

Gamarova, A. Yu., Mkrtichian, D. E., Rodriguez, E., Costa, V., \& Lopez-Gonzalez, M. J. 2003, PASPC, 292, 369

Garrido, R., Garsia-Lobo, E., \& Rodriguez, E. 1990, A\&A, 234, 262

Gáspár, A., Kiss, L. L., Bedding, T. R., et al., A\&A, 234, 262

Giuricin, G., Mardirossian, F., \& Mezetti, M. 1983a, ApJS, 52, 35

Giuricin, G., Mardirossian, F., \& Mezetti, M. 1983b, A\&AS, 54, 211

Giuricin, G., Mardirossian, F., \& Mezetti, M. 1984, A\&A, 131, 152

Heynderrickx, D., Waelkens, C., \& Smeyers, P. 1994, A\&AS, 105, 447

Kim, S.-L., Lee, J.-W., Youn, J.-H., Kwon, S.-G., \& Kim, C. 2002a, A\&Ap, 391, 213

Kim, S.-L., Kwon, S.-G., Youn, J.-H., Mkrtichian, D. E., \& Lee, J. W. 2002b, IBVS 5314

Kim, S.-L., Lee, J. W., Kwon, S.-G., et al. 2002c, IBVS No. 5325

Koch, R. H. 1960, AJ, 65, 139

Kusakin, A. V., Mkrtichian, D. E., \& Gamarova, A. Yu. 2001, IBVS No. 5106

Kürster, M., Schmitt, J. H. M. M., Custispoto, G., \& Dennerl, K. 1997, A\&A, 320, 831

Lehmann, H., \& Mkrtichian, D. E. 2004, A\&A, 413, 293

Maxted, P. F. L., Hill, G., \& Hilditch, R. W. 1994, A\&A, 282, 821

Mezzetti, M., Giuricin, G., \& Mardirossian, F. 1980, A\&A, 83, 217

Mkrtichian, D. E., \& Gamarova, A. Y. 2000, IBVS No. 4836

Mkrtichian, D. E., Kusakin, A. V., Gamarova, A. Y., et al. 2002a, PASPC, 256, 259

Mkrtichian, D. E., Kusakin, A. V., Gamarova, A. Y., \& Nazarenko, V. 2002b, PASPC, 259, 96 
Murdich, K. A., Hearnshaw, J. B., \& Clark, M. 1993, ApJ, 413, 349

Nather, R. E., \& Robinson, E. L. 1974, ApJ, 190, 637

Nelson, C. A., \& Eggleton, P. P. 2001, ApJ, 552, 664

Ohshima, O., Narusawa, S.-Y., Akazawa, H., et al. 1998, IBVS No. 4581

Ohshima, O., Narusawa, S.-Y., Akazawa, H., et al. 2001, AJ, 122, 418

Paczynski, B. 1971, ARA\&A, 9, 183

Popper, D. M. 1973, ApJ, 185, 265

Rodriguez, E., Gonzalez-Bedolla, S. F., Rolland, A., Costa, V., \& Lopez de Coca, P. 1997, A\&A, 324, 959

Rodriguez, E., Claret, A., Sedano, J. L., Garcia, J. M., \& Garrido, R. 1998, A\&A, 340, 196
Rodriguez, E., Lopez-Ganzalez, M. J., Rolland, A., Costa, V., \& Gonzalez-Bedolla, S. F. 2001, A\&A, 376, 489

Rodriguez, E., Garcia, J. M., Mkrtichian, D. E., et al. 2003, MNRAS, 347,1317

Sarma, M. B. K., Vivekananda Rao, P., \& Abhyankar, K. D. 1996, ApJ, 458, 371

Struve, O., \& Huang, S.-S. 1956, AJ, 61, 300

Ulrich, R. K., \& Burger, H. L. 1976, ApJ, 206, 509

Van Hamme, W., \& Wilson, R. E. 1990, AJ, 100, 1981

Van Hamme, W., \& Wilson, R. E. 1984, A\&A, 141, 1

Waelkens, C. 1996, A\&A, 311, 873

Watson, R. D. 1988, Astrophys. Space Sci., 140, 255 